\title{
Cytokinin but not gibberellin application had major impact on the phenylpropanoid pathway in grape
}

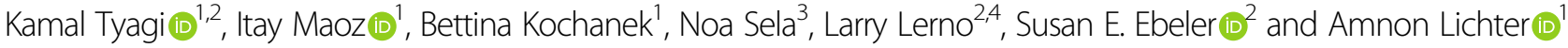

\begin{abstract}
Cytokinin and gibberellic acid (GA) are growth regulators used to increase berry size in seedless grapes and it is of interest to understand their effects on the phenylpropanoid pathway and on ripening processes. $G A_{3}$ and synthetic cytokinin forchlorfenuron ( $\mathrm{N}$-(2-chloro-4-pyridyl)-N'-phenylurea, CPPU) and their combination were applied to $6 \mathrm{~mm}$ diameter fruitlets of 'Sable Seedless', and berries were sampled 51 and 70 days (d) following application. All treatments increased berry size and delayed sugar accumulation and acid degradation with a stronger effect of CPPU. CPPU, but not GA, reduced berry color and the levels of anthocyanins. While CPPU reduced the levels of anthocyanins by more than $50 \%$, the combined treatment of GA+CPPU reduced the levels by about $25 \%$ at $51 \mathrm{~d}$. CPPU treatment had minor effects on flavonols content but increased the levels of monomeric flavan-3-ols by more than two-fold. Phloroglucinol analysis using HPLC showed that proanthocyanidin content was significantly increased by CPPU, whereas mean degree of polymerization was reduced from 26 to 19. Volatile analysis by GC-MS showed changes in composition with CPPU or GA treatment with potential impact on flavor. RNA-seq analysis showed that GA had a minor overall effect on the transcriptome whereas CPPU had pronounced effects on gene expression at both 51 and $70 \mathrm{~d}$. Comparing the control and CPPU at similar Brix of ca. $19.7^{\circ}$, a reduced expression of stilbene synthases (STSs) including their regulators MYB14 and MYB15, and other phenylpropanoid-related genes was observed in CPPUtreated grapes. Overall, our study shows that CPPU had a major influence on the phenylpropanoid pathway and affected multiple ripening-related processes.
\end{abstract}

\section{Introduction}

Fruit development is mediated by plant growth regulators (PGRs) that control its major developmental processes ${ }^{1}$. As grape berries develop, they exhibit a double sigmoid growth curve separated by veraison that marks the beginning of ripening ${ }^{2}$. Cell division and expansion are the major events during the first phase and are accompanied by synthesis and accumulation of organic acids, methoxypyrazines, and phenolic compounds such as proanthocyanidins (PAs) and hydroxycinnamates. This phase of berry growth is under the control of auxins, gibberellins, and cytokinins. Auxins and gibberellins are

Correspondence: Amnon Lichter (vtlicht@agri.gov.il)

${ }^{1}$ Department of Postharvest Science, Agricultural Research Organization, The Volcani Center, Rishon LeZion, Israel

${ }^{2}$ Department of Viticulture and Enology, University of California, Davis, CA 95616, USA

Full list of author information is available at the end of the article mostly produced by the seeds while the source of cytokinins in fruits is less established and it is likely to be imported from the plant ${ }^{2}$. The number of seeds in the berry can determine berry size, and the lack of seeds or the presence of unfertilized ovules in sternospermocarpic ('seedless') berries can be partly complemented by external gibberellin ${ }^{3}$. Schematic representation of the levels of PGRs during development suggests that auxin levels are high at berry set and decrease during phase 1 while cytokinins and gibberellins peak during phase $1^{2}$. The second phase, verasion, marks the beginning of major processes in grapes ripening, berry softening and anthocyanins accumulation in colored varieties. There are reports of a small peak of ethylene preceded by a large peak of abscisic acid (ABA) which coincide with veraison ${ }^{4}$. Brassinosteroids increase at veraison and participate in ripening, possibly by modulation of ethylene content ${ }^{4}$. On the other hand, auxin treatments retard sugar and

\section{(c) The Author(s) 2021}

(c) (i) Open Access This article is licensed under a Creative Commons Attribution 4.0 International License, which permits use, sharing, adaptation, distribution and reproduction cc) in any medium or format, as long as you give appropriate credit to the original author(s) and the source, provide a link to the Creative Commons license, and indicate if changes were made. The images or other third party material in this article are included in the article's Creative Commons license, unless indicated otherwise in a credit line to the material. If material is not included in the article's Creative Commons license and your intended use is not permitted by statutory regulation or exceeds the permitted use, you will need to obtain permission directly from the copyright holder. To view a copy of this license, visit http://creativecommons.org/licenses/by/4.0/. 
anthocyanin accumulation and prevent the decrease in acidity and chlorophyll concentration, and also cause a delay in the usual ripening-associated increase in the levels of $\mathrm{ABA}^{5}$. The third phase in berry development is ripening which is characterized by accumulation of glucose and fructose, as well as a decrease in the levels of organic acids. Volatile compounds that are produced by grape berries during development and ripening include fatty acid derivatives that are the most abundant group, monoterpenes that are prominent flavor compounds in Muscat flavored grapes, sesquiterpenes, $\mathrm{C}_{13}$ norisoprenoids, volatile sulfur compounds, and methoxypyrazines $^{4,6,7}$. Some volatile compound types such as methoxypyrazines and sesquiterpenes, $C_{13}$ norisoprenoids and volatile fatty acid derivatives accumulate in the berry before veraison while volatile monoterpenes and volatile sulfur compounds accumulate during ripening ${ }^{4,5}$.

Physiological studies on the role of PGRs in fruit development often rely on external applications of the hormones or their agonist followed by observations of the changes in fruit development. In table grapes there is a plethora of applicative studies aimed at increasing the berry size in 'seedless' varieties and the color of red varieties grown in hot regions ${ }^{8}$. The effect of GA was studied from the late $1950 \mathrm{~s}^{9}$ with timing and concentration being major factors. In 'seedless' grapes, application of GA to increase berry size is performed at a fruitlet diameter of 4-6 $\mathrm{mm}$ because earlier application can have negative impacts on fruit-set and berry shot (berry set too high and presence of very small berries) and later application is less effective. The effective concentration of GA can vary markedly according to sensitivity of the variety to the hormone. For example, 'Thompson Seedless' requires multiple applications and large cumulative amounts of GA exceeding $100 \mathrm{ppm}$, while a single application of 10 ppm of GA can triple the size of 'Black Finger' berries ${ }^{10}$. GA is also known to potentially delay maturity, increase pedicel thickness, and increase berry abscission depending on the application time. If applied on the whole vine on sensitive varieties, GA can also harm reproductive meristems and reduce subsequent yield. The molecular aspects of the synthesis, signal perception, and transduction of GA in grapes have been reported ${ }^{11,12}$.

The study of the effects of cytokinins on grape development focused largely on forchlorfenuron ( $\mathrm{N}$-(2-chloro4-pyridyl)- $\mathrm{N}^{\prime}$-phenylurea), a synthetic cytokinin known as CPPU, that has been tested and applied to regulate fruitset, size, and shape in several fruit crops ${ }^{13,14}$. In grapes, CPPU was also reported to delay maturation and reduce berry skin color, increase berry pedicel thickness and rigidity, increase cuticle content, and reduce weight loss of the rachis ${ }^{15-18}$. CPPU is commercially applied at levels of $5 \mathrm{ppm}$ or lower due to its potential adverse effects on maturation and postharvest quality. The time frame for application of CPPU is usually similar to that of GA and often in combination with GA at reduced concentrations ${ }^{16}$. Other cytokinins such as benzyl adenine had similar effects but a concentration of 500-1000 ppm was required to increase berry size ${ }^{19}$.

Phenylpropanoids are a large class of plant secondary metabolites derived from aromatic amino acids, phenylalanine in most plants or tyrosine in some monocots. The main branches of the phenylpropanoid pathway include lignans and lignins, stilbenes, coumarins, isoflavonoids, flavonoids, and $\mathrm{PAs}^{20}$. The biosynthesis of PAs, anthocyanins, and flavonols share common steps in the flavonoid pathway ${ }^{4}$. In grape berries, the first committed steps in PA biosynthesis are catalyzed by leucoanthocyanidin reductase (LAR) and anthocyanidin reductase (ANR) by converting anthocyanidins to flavan-3-ols such as $(+)$-catechin $(\mathrm{C})$ and (-)-epicatechin (EC), respectively. The resulting (-)-epicatechin and $(+)$-catechin derivatives can be oxidized to quinones, which are polymerized. However, it is not clear whether this polymerization of the soluble precursors proceeds enzymatically by laccases or non-enzymatically ${ }^{21}$. The subunits of PA are derived from 2,3-cis-flavan-3-ols (i.e., (-)-epicatechin and (-)-epigallocatechin (EGC), as well as from 2,3-trans-flavan-3-ols (i.e., (+)-catechin and (+)-gallocatechin) and are most commonly linked via $4 \beta \rightarrow 8 \mathrm{C}-\mathrm{C}$ bonds ${ }^{21}$. The PAs are oligomeric and polymeric flavan-3-ols that can range in size from 2 to 30 or more subunits. The regulation of the phenylpropanoid pathway was studied extensively in grapes with respect to anthocyanins, PAs, and other branches of the pathway with emphasis on the role of R2R3-MYB, bHLH, and WD40 transcription factors and their target genes ${ }^{4,5}$.

Our previous study demonstrated that CPPU causes a marked increase in tannin content of Thompson Seedless $^{22}$. Thompson Seedless is a major variety but it does not produce anthocyanins and is very low in volatile content. In the current study we presented the following questions: (1) how do CPPU and GA affect tannin accumulation in a variety that is rich in anthocyanins and volatile compounds; and (2) how are biological processes at ripening affected by the treatments. These questions were addressed at the phenological level, by the analysis of relevant metabolites from the phenylpropanoid pathway, by volatile composition, and by transcriptome analysis.

\section{Materials and methods}

\section{Plant material and tissue collection}

Experiments were carried out on Vitis vinifera cv. Sugrasixteen (SABLE SEEDLESS ${ }^{\circledR}$ ) (Sun World, Bakersfield, CA) that will be referred as 'Sable'. Vines of 'Sable' were grafted on Richter rootstock that was 7 year old and grown in Israel in the Lachish area (lat. $31^{\circ} 33^{\prime}$, long. $34^{\circ}$ $\left.51^{\prime}\right)$. All viticulture practices were performed as describe 
previously $^{23}$. The experimental plot comprised of four replications of three vines each, arranged in a randomized block design. Clusters were manually sprayed to full wetness with the growth regulators gibberellic acid $\left(\mathrm{GA}_{3}\right.$, ProGibb 40, Valent BioScience, Walnut Creek, CA, USA) and forchlorfenuron (CPPU, a synthetic cytokinin, SKW, Wittenberg, Germany) in a concentration of 20 and $5 \mathrm{ppm}$, respectively, with $0.025 \%$ Triton-X100 (Adama Agan, Ashdod, Israel) as a wetting agent. A combination of GA and CPPU (hereafter GA + CPPU) was also sprayed on the berry at the same concentrations. 'Sable' was treated on 14 May 2018 at the berry diameter of $6.0 \pm$ $0.08 \mathrm{~mm}$ (E-L stage of 31$)^{24}$. Phenological data for 'Sable' was collected at the time of treatment (i.e., day 0 ), and 7 , 34,51 , and 70 days (d) post treatment $(34,51$, and $70 \mathrm{~d}$ corresponding to stages $35,36-37$, and 38 of the modified E-L scale, respectively). Sampling for metabolites, volatiles, and gene expression was carried out at 51 and $70 \mathrm{~d}$ after treatment, the time points which represent the beginning and end of commercial harvest. Sampling at all time-points was of 90 berries pooled from 20 to 30 clusters randomly collected from the four vineyard replications. For metabolite and RNA-seq analysis, a disk of $14-16 \mathrm{~mm}$ was removed along the longitudinal axis of each berry and was frozen in liquid nitrogen. The berry disks were homogenized in three replicates of 30 disks each using an IKA homogenizer (IKA, Staufen, Germany) with liquid nitrogen and were stored at $-80^{\circ} \mathrm{C}$ for further analysis. The remaining part of the berries was used for measurement of total soluble solids (TSS) and titratable acidity (TA).

\section{Measurement of total soluble solids, titratable acidity, and tasting}

Measurements of TSS and TA in 'Sable' berries of GA, CPPU, and GA + CPPU treatments were carried out as previously described ${ }^{23}$. The juice was obtained by using a fruit juicer (EF-800, Sachs, Tel Aviv, Israel) from 30 berries. TSS was determined by a digital refractometer (Atago, Tokyo, Japan) and denoted as ${ }^{\circ}$ Brix. TA was measured by means of titration with $0.1 \mathrm{~N} \mathrm{NaOH}$ to $\mathrm{pH}$ 8.2 with a Metrohm automatic titrator (Herisau, Switzerland) and expressed as tartaric acid equivalents. All the above analyses were performed on fresh samples at the harvest date. Informal tasting was done by three expert tasters that scored the samples in hedonic scale of 1-9 (9 being excellent taste).

\section{Anatomical observation of 'Sable' berry skin}

Sample preparation and microscopic examination of 'Sable' berries at $33 \mathrm{~d}$ after GA, CPPU, and GA + CPPU treatment were performed as described by Tyagi et al. ${ }^{23}$. Briefly, transverse hand sections from 'Sable' berries of GA, CPPU, and GA + CPPU treatment were immediately immersed in a solution ( $\mathrm{v} / \mathrm{v})$ containing formaldehyde, acetic acid, ethanol, and water in ratios of 10: 5: 50: 35, respectively. After fixation, tissue sections were serially diluted by ethanol and subsequently a stepwise exchange of ethanol with Histoclear (xylem substitute) was carried out. Samples were embedded in paraffin and cut with a microtome (Leica RM2245, Leica Biosystems, Nussloch, Germany) into $12 \mu \mathrm{m}$ thick sections. Sections were stained with Safranin O that stains nuclei, lignified suberized, or cutinized cell wall in red, and with fast green FCF that stains cellulose in green-blue ${ }^{25}$ and sections were examined under a light microscope (Leica DMLB).

\section{Extraction and analysis of phenolic compounds by HPLC}

Extraction and analysis of phenolics from mature berries (GA, CPPU, and GA + CPPU) of 'Sable' (51 and $70 \mathrm{~d}$ after treatment) were carried out as described previously $^{23}$. Briefly, anthocyanins and other monomeric phenols were extracted using $0.8 \mathrm{~g}$ of frozen powder and homogenized in $3 \mathrm{~mL}$ of $50 \%$ (v/v) methanol (containing $0.1 \% \mathrm{v} / \mathrm{v}$ hydrochloric acid and $0.1 \% \mathrm{w} / \mathrm{v}$ ascorbic acid). Homogenates were mixed and incubated at $4{ }^{\circ} \mathrm{C}$ for $4 \mathrm{~h}$ on ice with intermittent mixing and were centrifuged at $4000 g$ (Eppendorf, Hamburg, Germany) for $5 \mathrm{~min}$. Pellets from the above samples were re-extracted twice and all the supernatants were pooled to make the final volume of $5 \mathrm{~mL}$. Of this, $1 \mathrm{~mL}$ of supernatant was used for the analysis of anthocyanins and other monomeric phenols and the remaining $4 \mathrm{~mL}$ was stored at $-20^{\circ} \mathrm{C}$. The pellet of the above extract was used further for analysis of PAs. The anthocyanins and other monomeric phenols of 'Sable' berries of GA, CPPU, and GA + CPPU were analyzed by Agilent Infinity series 1260 RP-HPLC (Agilent Technologies, Santa Clara, CA, USA). All data processing and analysis were accomplished using Agilent CDS ChemStation software on an Agilent 1260 Infinity HPLC (Agilent Technologies).

\section{PA extraction, purification, and phloroglucinol analysis}

PA extraction, purification, phloroglucinolysis, and data analysis were performed as described by Tyagi et $\mathrm{al}^{23}$. Briefly, the pellet from the previous section (monomeric phenolics extraction for 'Sable' 51 and $70 \mathrm{~d}$ after GA, CPPU, and GA + CPPU treatment) was vortexed in $8 \mathrm{~mL}$ of $70 \%$ acetone containing $0.1 \%$ ascorbic acid $(\mathrm{w} / \mathrm{v})$ and $0.05 \%$ trifluoroacetic acid (v/v) for the extraction of larger polymers. After overnight extraction at $4{ }^{\circ} \mathrm{C}$ with occasional mixing, the acetone extract was centrifuged at $4000 \mathrm{~g}$ and the pellet was re-extracted twice with acidified acetone to make a final volume of $12 \mathrm{~mL}$. The methanol supernatant $(4 \mathrm{~mL}$ extract from anthocyanins and monomeric phenols) and acetone supernatant were pooled and reduced under vacuum to near dryness at $34{ }^{\circ} \mathrm{C}$ using a Buchi ${ }^{\circledR}$ Rotavapor R110 (Flawil, Switzerland) 
and the extract was dissolved in $1 \mathrm{~mL}$ with methanol. PAs were purified by solid phase extraction (SPE) using bed columns containing $10 \mathrm{~mL}$ of Toyopearl HW-40F sizeexclusion media (Sigma-Aldrich, USA) and the eluates from the SPE columns containing isolated PA were concentrated under reduced pressure, dissolved in $500 \mu \mathrm{L}$ methanol, and stored at $-80^{\circ} \mathrm{C}$ until analysis. The composition of isolated PA from 'Sable' berries was determined by phloroglucinolysis and the reaction products were chromatographically separated by RP-HPLC on an Agilent Infinity series 1260 HPLC system (Agilent Technologies) equipped with a DAD detector using a binary solvent gradient.

\section{GC-MS extraction and analysis of free volatiles}

Volatiles from mature berries of 'Sable' ( 51 and $70 \mathrm{~d}$ in GA, CPPU, and GA + CPPU after treatment) were extracted and analyzed according to the procedure described $^{26}$. Volatiles were extracted from $2 \mathrm{~g}$ of frozen powder in three replicates. Volatile compounds were analyzed using MSD Chemstation E.02.00.493, and their MS description was based on the NIST library, Version 05 (Agilent Technologies, USA). Volatile compounds were identified based on the Retention Index (RI) and mass spectra, specific compounds were also identified by authentic standards as described previously ${ }^{27}$.

\section{RNA extraction, sequencing, and data analysis}

Total RNA extraction and DNase treatment from $1.2 \mathrm{~g}$ of frozen powder of 'Sable' (51 and $70 \mathrm{~d}$ after treatment) in control (Ctrl), GA-, and CPPU-treated berries was performed as described previously ${ }^{23}$. RNA was sequenced by the DNA Technologies Core facility (University of California, Davis, CA, USA). Gene expression profiling was performed using a 3'-Tag-RNA-Seq protocol. Barcoded sequencing libraries were prepared using the QuantSeq FWD kit (Lexogen, Vienna, Austria) for multiplexed sequencing using both the UDI-adapter and UMI SecondStrand Synthesis modules (Lexogen). The preparation followed the recommendations of the manufacturer with two modifications, first being a reduction in fragmentation time to $10 \mathrm{~s}$ at $85^{\circ} \mathrm{C}$ and the second was extending the reverse transcription step to $1 \mathrm{~h}$ at $42^{\circ} \mathrm{C}$. The fragment size distribution of the libraries was confirmed via microcapillary gel electrophoresis on a LabChip GX system (PerkinElmer, Waltham, MA, USA). The libraries were quantified by fluorometry on a Qubit fluorometer (Life Technologies, Carlsbad, CA, USA), and combined in equimolar ratios. The library pool was quantified via $\mathrm{qPCR}$ with a Kapa Library Quant kit (Kapa Biosystems/Roche, Basel, Switzerland) on a QuantStudio 5 system (Applied Biosystems, Foster City, CA, USA). Up to 48 libraries were sequenced per lane on a HiSeq 4000 sequencer (Illumina, San Diego, CA, USA) with single-end 100 bp reads.
The raw reads of 18 libraries-three biological replications for each of the three treatments, Ctrl, GA, and CPPU at 51 or $70 \mathrm{~d}$-were subjected to quality filtering and adapter removal by HTStream software (https:// github.com/s4hts/HTStream). Cleaned sequences were mapped to the genome version Vcost.3 of $V$. vinifera downloaded from the site (https://urgi.versailles.inra.fr/ Species/Vitis/Data-Sequences). STAR software was used to align clean reads to the reference genome. Sequence data was deposited to BioProject at NCBI under the accession PRJNA686849. Bioconductor DESeq2 was used to identify differentially expressed transcripts, based on the count estimates for each transcript. Transcripts were regarded as differentially expressed if they reached the threshold of FDR $<0.05$ and the $\log 2$-fold change was $<-1$ or $>1$. GO enrichment analysis was done using Blast2Go. KEGG pathway enrichment was done using the KOBAS 3.0 server (http://kobas.cbi.pku.edu.cn/). The expression data was normalized by using the trimmed mean of $M$ values method (TMM). For the clustering we used cutree in $\mathrm{R}$ that takes as parameters $k$ number and cut the hierarchical clustering tree into $k$ clusters (https:// www.rdocumentation.org/packages/stats/versions/3.6.2/ topics/cutree).

\section{Statistical analysis and data representation}

A minimum of three biological replicates were used for each experiment and all results were expressed as mean \pm $\mathrm{SD}$ (standard deviation) or as specified in the figure legends. Each biological replicate consisted of 30 berries. Statistical analysis was done by ANOVA followed by Tukey post-hoc tests or Student $t$-tests as indicated by JMP (ver. 13.0; SAS Institute, Cary, NC, USA). Principal component analysis (PCA) and variables importance in projection (VIP) score were performed to obtain overall clustering of the samples from the volatile analysis, using Metaboanalyst $4.0^{28}$. Venn diagrams were performed by the free online BEG tool (http://bioinformatics.psb.ugent.be/webtools/Venn/). Figures were prepared with $\mathrm{R}$ packages ggplot2, tidyverse, agricolae, svDialogs, and multcompView.

\section{Results \\ Effect of early GA and CPPU treatment on 'Sable' berry properties along development}

Gibberellin and cytokinin applied at fruit-set are known to increase berry size, but due to the complexity of agricultural systems, the intensity of the response can vary among seasons. The PGR treatments were performed at a fruitlet size of $6.0 \pm 0.08 \mathrm{~mm}$ on 'Sable Seedless'. Fruitlet diameter and weight increased significantly by application of both GA and CPPU measured $7 \mathrm{~d}$ after treatment (Fig. 1a, c). Interestingly, at $34 \mathrm{~d}$ post treatment, berry diameter and weight of CPPU-treated berries was smaller than the control but this trend was reversed at later time 


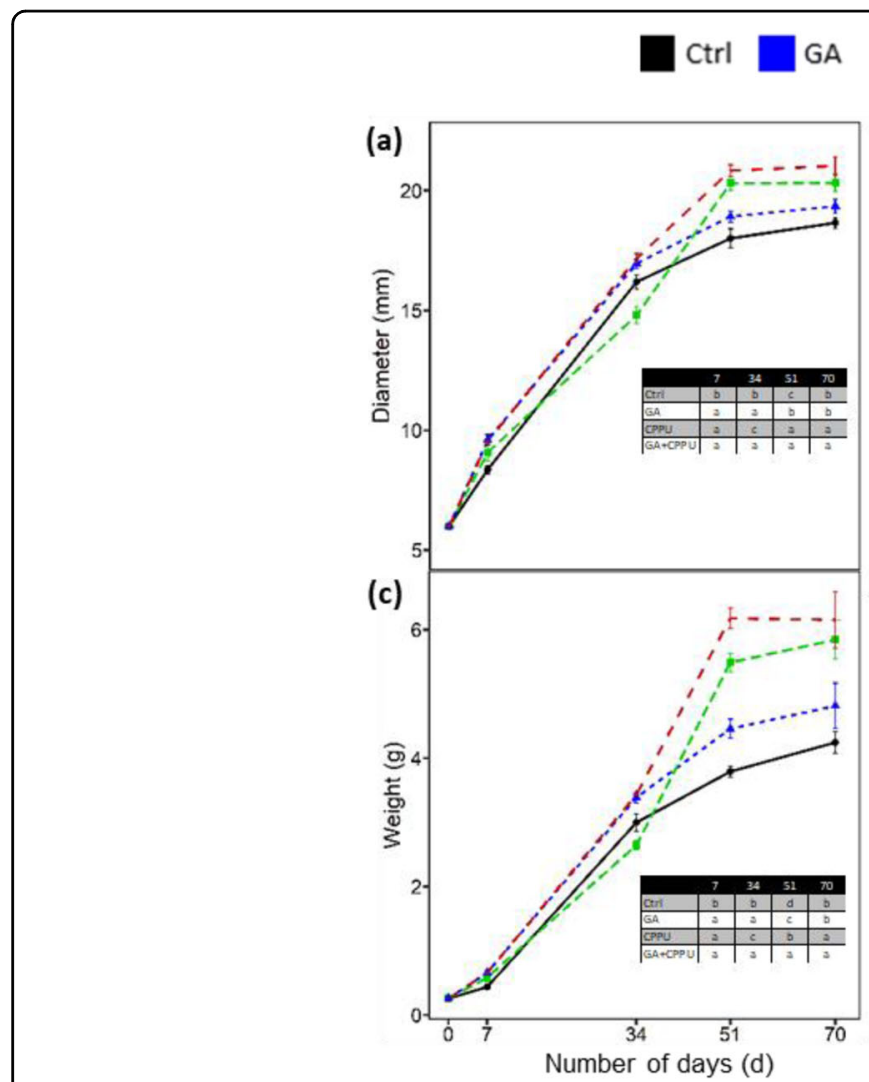

(e)

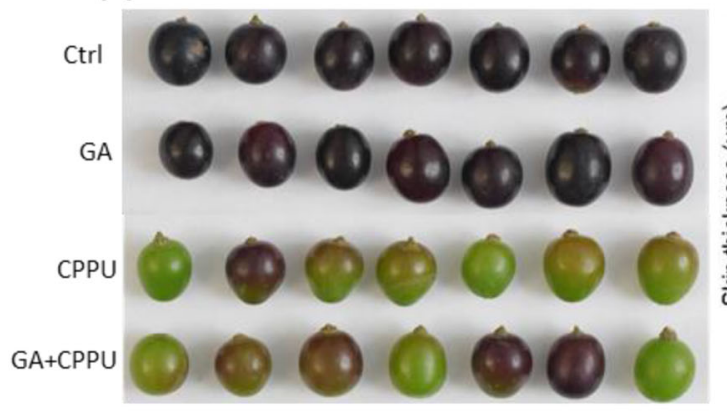

(f)

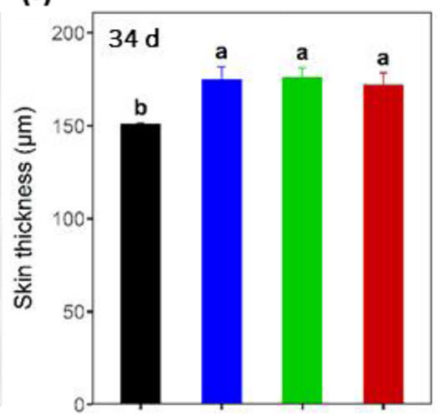

(g)

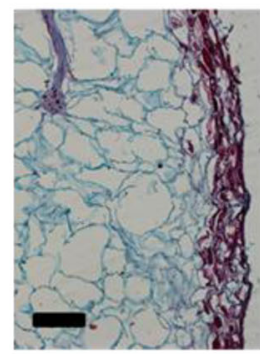

Control

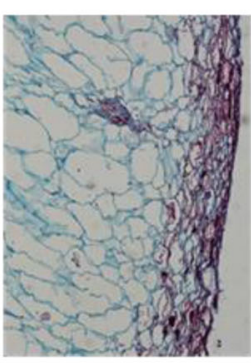

GA

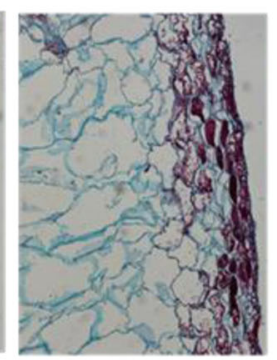

CPPU

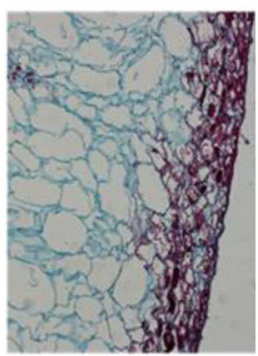

$\mathrm{GA}+\mathrm{CPPU}$

Fig. 1 Effect of growth regulator treatments after berry set on berry properties during development and ripening in 'Sable'. Berries were treated with GA, CPPU, and GA + CPPU after the berry set. a Diameter ( $\mathrm{mm}), \mathbf{b}{ }^{\circ}$ Brix, $\mathbf{c}$ weight ( $\mathrm{g}$ ), $\mathbf{d}$ titratable acidity (\% TA), e berry appearance $34 \mathrm{~d}$ after treatment, $\mathbf{f}$ berry skin thickness $(\mu \mathrm{m})$, and $\mathbf{g}$ representative berry sections stained with Safranin, from the Ctrl, GA, CPPU, and GA + CPPU $34 \mathrm{~d}$ after treatments. (a-d) Sample size was of 30 berries and values are the mean and standard deviation of three replications and the table in the panel indicates if the differences among treatment are significant by Tukey post-hoc test at $p \leq 0.05$. Value in panel (f) are mean and standard deviation of 9 sections from 3 berries and different letters above each bar denote significant differences between the treatments. The horizontal black bar represents a size of $100 \mu \mathrm{m}(\mathbf{g})$ 
points. At 51 and $70 \mathrm{~d}$ after the treatments, CPPU increased berry weight and diameter significantly with respect to the control and the GA treatment. The GA treatment increased berry size by $17.5 \%$ and $13.4 \%$ at 51 and $70 \mathrm{~d}$, respectively, but at a later time point, the difference was not statistically significant from the control. The diameter and weight of berries treated with the combined treatment of GA + CPPU was higher than that of CPPU alone at both 51 and $70 \mathrm{~d}$. Brix of the control grapes was higher at all time-points followed by GA and CPPU (Fig. 1b). At 34 d, berries treated with GA + CPPU had higher Brix as compared to CPPU-treated berries, but at later time points there were no differences among the two treatments. CPPU had a major effect on TA that was much higher than the other treatments at $34 \mathrm{~d}$ after treatment (Fig. 1d). At both 34 and $51 \mathrm{~d}$, GA seemed to mitigate the effect of CPPU on TA. With respect to appearance of the berries $34 \mathrm{~d}$ after the treatment, there was a clear delay in color development by CPPU and the treatment of GA + CPPU (Fig. 1e). Transverse sections of the berries showed that the epidermal layer was thicker either in both GA- and CPPU-treated berries or the combined treatment as compared to the untreated control (Fig. 1f, g). Informal tasting done by experts indicated excellent taste rated as 8 with multiple aromatic notes for the control and GA treatments, while in berries from the CPPU treatment there was some astringency that reduced the score to 7.5 or 7.0 for the combined treatment.

\section{CPPU but not GA treatments alter the levels of flavonoid compounds}

The cytokinin CPPU was shown to increase total tannins in Thompson Seedless that bears green berries ${ }^{22}$. It was therefore of interest to determine what effect CPPU has on black berries and if GA has similar effects. HPLC analysis was carried out for glucosides, acylated and coumaroylated forms of delphinidin, cyanidin, petunidin, peonidin, and malvidin (Fig. 2a and Table S1). CPPU and GA + CPPU treatments reduced the levels of the majority of these compounds at both 51 and $70 \mathrm{~d}$ as compared to the control. Quantitatively, CPPU reduced the levels of the anthocyanin glucosides by ca. $50 \%$ at both time points. The GA + CPPU treatment reduced the levels of the anthocyanin glucosides to ca. $75 \%$ and $53 \%$ at 51 and $70 \mathrm{~d}$, respectively, as compared to the control. At $51 \mathrm{~d}$ post treatment, GA reduced the inhibitory effect of CPPU but this effect was not maintained at $70 \mathrm{~d}$ (Fig. 2a). Anthocyanin glucosides are the major form present in 'Sable' comprising $63-71 \%$ of the total anthocyanins and the remaining are acetylated and coumaroylated forms (Table 1). Interestingly, CPPU reduced the proportion of the glucoside forms at both 51 and $70 \mathrm{~d}$ and this decrease was accompanied by an increase in coumaroyl glucosides at both time points and acetylated glucosides at $70 \mathrm{~d}$. GA had an intermediate effect on the proportion of the anthocyanin forms.

Flavonols are an important branch of the flavonoid pathway and therefore it was of interest to determine the effect of the treatments on major grape flavonols (Fig. 2b). Myrcetin glycoside was the major flavonol detected with an average of $81-89 \%$ among the compounds tested. While variation among replications was significant, two trends are worth noting. At $51 \mathrm{~d}, \mathrm{CPPU}$ alone or in combination with GA reduced the levels of flavonols to ca. $60 \%$ of the control. At $70 \mathrm{~d}$ there was an increase in levels of the flavonols and decrease in the effect of CPPU. Quercetin galactoside did not significantly differ among the treatments.

Flavan-3-ols are the building blocks of the PA chains that are synthesized in the early stages of berry development. Figure $2 \mathrm{c}$ displays the three major flavan-3-ols: $(+)$-catechin, (-)-epicatechin (EC), and (-)-epigallocatechin (EGC). Clearly, CPPU-treated berries contained more flavan-3-ols and their level increased during ripening. At the late sampling, GA + CPPU reduced the level of the flavan-3-ols as compared to the CPPU treatment alone. The hydroxycinnamic acid derivatives, caffeic acid, caftaric acid, coutaric acid, and ferulic acid changed in different ways: caftaric acid was highest in the early stage in CPPU-treated berries; caffeic acid levels were reduced by CPPU at the early stage and it was absent in the late harvest; coutaric acid levels were induced by CPPU and reduced by the combined treatment relative to the control; the levels of ferulic acid were low but increased with ripening without a distinct pattern (Table S1).

\section{CPPU increases PA levels but shortens the polymer length in berries}

To further investigate the effect of GA, CPPU, and GA + CPPU on PA composition, phloroglucinolysis was performed and differences were monitored using HPLC (Figs. 2d-f, S1, and Table S2). Phloroglucinolysis hydrolyzes the polymeric PAs generating terminal units (C, EC, and ECG (epicatechin gallate)) and extension units with phloroglucinol adduct (labeled by $-\mathrm{P}$ ). CPPU increased the levels of C, EC, EC-P, and EGC-P. GA did not affect the levels of the PA monomers while the treatment with GA + CPPU reduced the levels of C, ECG, EC-P, and EGC-P only at $70 \mathrm{~d}$ after the treatments (Fig. S1). Monomeric PAs did not change with ripening with the exception of ECG that was lower at $70 \mathrm{~d}$ compared to the control treatment. The total PA level clearly shows the effect of CPPU and also shows that the combined treatment reduced the level of PAs at $70 \mathrm{~d}$ in agreement with the data on the free monomers (Fig. 2c). The treatments with GA or CPPU had minor effects on the \% galloyl units but the combined treatment reduced their level. The mean degree of polymerization (mDP) was significantly 


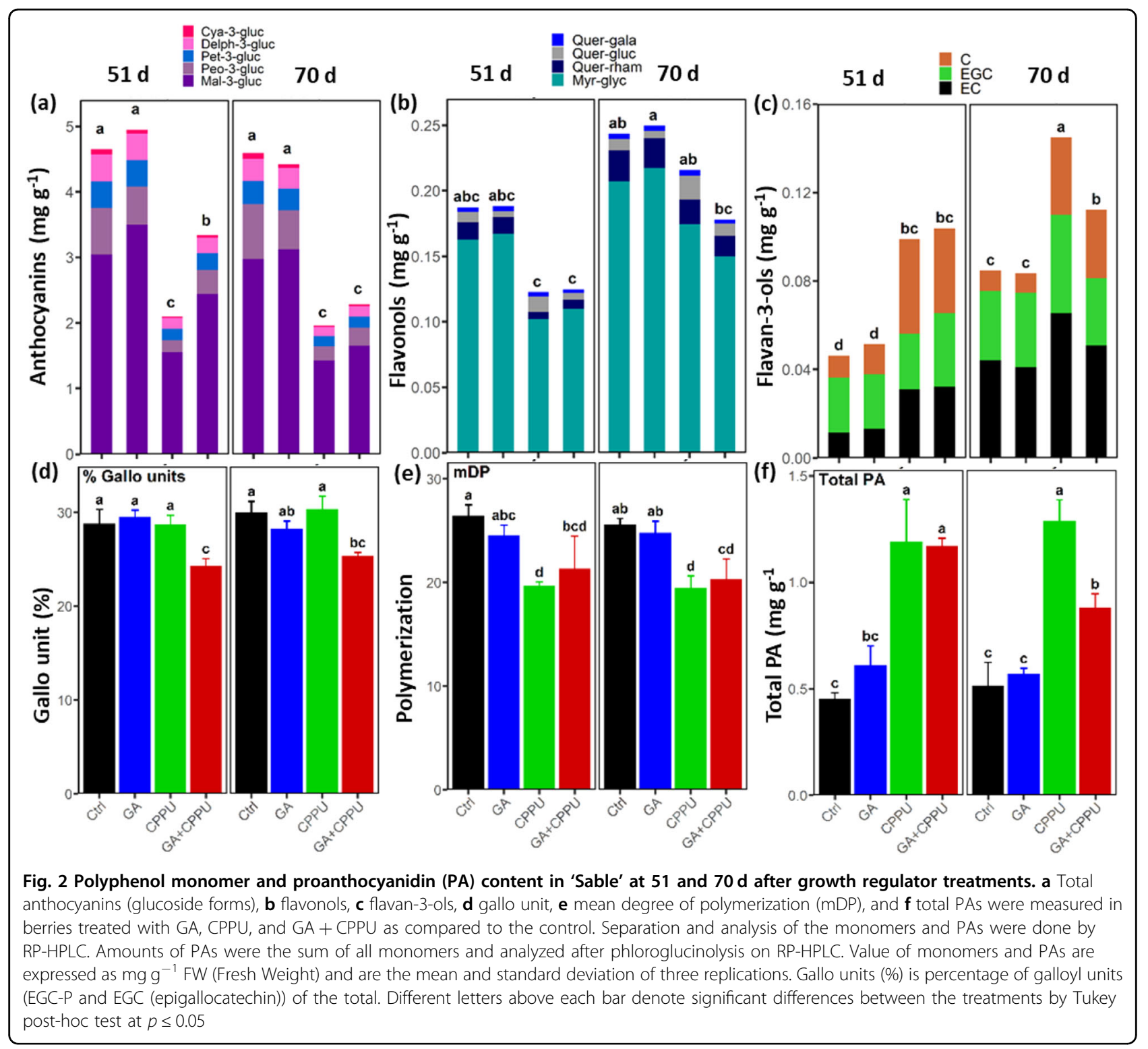

Table 1 Influence of GA, CPPU, and GA + CPPU treatments on 'Sable' proportion (\%) of glucoside, acetylated and coumaroylated anthocyanin forms at 51 and $70 \mathrm{~d}$ after treatment

\begin{tabular}{|c|c|c|c|c|c|c|c|c|}
\hline \multirow[t]{2}{*}{ Anthocyanin forms } & \multicolumn{4}{|l|}{$51 \mathrm{~d}$} & \multicolumn{4}{|l|}{$70 \mathrm{~d}$} \\
\hline & Ctrl & $\mathrm{GA}$ & CPPU & $\mathrm{GA}+\mathrm{CPPU}$ & Ctrl & $\mathrm{GA}$ & CPPU & $\mathrm{GA}+\mathrm{CPPU}$ \\
\hline Gluc & $71.8 \pm 0.17$ & $69 \pm 0.74$ & $67.9 \pm 0.45$ & $67.7 \pm 0.12$ & $68.7 \pm 0.48$ & $66.9 \pm 0.32$ & $63.4 \pm 0.18$ & $64.7 \pm 0.49$ \\
\hline Acetylgluc & $8.2 \pm 0.03$ & $8.4 \pm 0.15$ & $8.3 \pm 0.05$ & $8.4 \pm 0.08$ & $7.5 \pm 0.1$ & $8.4 \pm 0.14$ & $9.3 \pm 0.18$ & $9 \pm 0.07$ \\
\hline Coumgluc & $20 \pm 0.16$ & $22.6 \pm 0.59$ & $23.8 \pm 0.41$ & $23.9 \pm 0.04$ & $23.7 \pm 0.45$ & $24.7 \pm 0.2$ & $27.4 \pm 0.31$ & $26.3 \pm 0.43$ \\
\hline
\end{tabular}

Different anthocyanin form are anthocyanin-3-O-glucoside (Gluc), anthocyanin-3-O-acetylglucoside (Acetylgluc), and anthocyanin- 3-O-(6-p-coumaroyl) glucoside (Coumgluc). Values in bold and italic differ significantly from the control according to Student $t$-test at $p \leq 0.05$ and $n=3$ 
lower in CPPU and GA + CPPU treatments as compared to the control (ca. $26 \mathrm{mDP}$ in the control versus ca. $20 \mathrm{mDP}$ in the treatments).

\section{Volatile compounds affected by the growth regulator treatments}

To evaluate the effect of GA, CPPU, and GA + CPPU treatment at berry set on the volatiles composition, berries of 'Sable' were sampled 51 and $70 \mathrm{~d}$ after the treatments. GC-MS analysis allowed identification of 58 (51 d) and $67(70 \mathrm{~d})$ volatile compounds with quantitative data calculated relative to the internal standard (Table S3). Analysis of the differences in volatile content by ANOVA showed that 62 of the 68 compounds present at both time points differed in content by treatments or time (Table S4). PCA indicated strong separation of the volatile compounds according to the harvest time with $\mathrm{PC} 1$ at $49.6 \%$ (Fig. 3a). PC2 contributed $26.5 \%$ of the total variance and reflected the effect of CPPU at $51 \mathrm{~d}$, but at $70 \mathrm{~d}$ there was convergence of the variance and overall differences among the treatments seemed insignificant. VIP score analysis for the volatile compounds at $51 \mathrm{~d}$ pointed to 15 compounds having significant scores (Fig. 3b). CPPU suppressed the levels of the monoterpenes, $\alpha$-terpineol, linalool, and limonene, as well as the carotenoid derivative, $\beta$-cyclocitral and the fatty acid derivative, hexanal. However, GA + CPPU increased the levels of these compounds. The levels of 2-hexenal $(\mathrm{Z})$ and pentanal were higher in CPPU-treated berries while GA increased the level of methyl geranate. At $70 \mathrm{~d}$, CPPU increased and GA or GA + CPPU decreased the levels of decanal and 5 other compounds (Fig. 3c). In contrast, GA increased the levels of trans and cis-rose oxide, $\beta$-bourbonene, and methyl geranate. Decanal, methyl geranate, ethyl acetate, and $\alpha$-terpineol were common among the two time points and the first two compounds showed the same pattern.

\section{CPPU but not GA had long-term effects on gene expression in berries}

PGRs may have multiple immediate effects on gene expression, but, in this study, we were interested in understanding the long-term effects of GA and CPPU on the berries at harvest. To this end, RNA-seq was carried out on 'Sable' berries at 51 and $70 \mathrm{~d}$ after the treatments, the time points that can be defined as early and late ripening with respect to the untreated control. A total of 3801 differentially expressed genes (DEGs) among all treatments were identified (Table S5). Venn diagram analysis of control vs. GA showed that there were hardly any differences between the control and GA treatments either at the early or late ripening, but there were significant differences in gene expression among the ripening stages (Fig. 4a). In contrast, the CPPU treatment imposed significant differences on gene expression at either the early or late stages of ripening (Fig. 4b). Six main clusters representing gene expression patterns were identified (Fig. 4c). KEGG pathway enrichment showed that only cluster 3 and cluster 4 contained significantly enriched pathways (Table 2). Six out of the 13 processes related to cluster 3 were assigned to the phenylpropanoid pathway. The first process registered for cluster 3 was 'circadian rhythms' but analysis of the relevant KEGG map suggested that all the 20 enriched genes in this bin were actually family members of chalcone synthase (CHS) which is a central flavonoid pathway. The 'ubiquinone and other terpenoid-quinone biosynthesis' bin included $4 C L$, trans-cinnamate 4-monooxygenase, and isochorismate synthase. Other bins of cluster 3, excluding the large unspecific bins of 'Biosynthesis of secondary metabolites' and 'Metabolic pathways', will be discussed later. Cluster 4 contained 2 out of 5 bins assigned to 'photosynthesis' or 'photosynthesis - antenna proteins'. The genes in these bins belonged to photosystem I, photosystem II, cytochrome b6/F complex, and LHC1 and LHC2 complexes. In the 'Steroid biosynthesis' bin, genes included squalene synthase, squalene monoxygenase, and other sterol biosynthesis genes upstream of brassinosteroid biosynthesis.

\section{Comparison of the effects of CPPU on gene expression at similar Brix}

Because GA did not contribute a significant number of genes to the DEG pool, it was eliminated from further analysis. There were major differences in ripening between the untreated grapes and grapes treated with CPPU, and this was also expressed in the number of DEGs at both early and late sampling. At the early and late sampling, there were 697 and 382 DEGs between the untreated and the CPPU-treated berries, respectively, and the reduction in the number of DEGs was mainly in the late sampling for genes with lower expression following CPPU treatment (Tables S6 and S7). KEGG analysis of these two groups did not show any functional bin enrichment. In a separate analysis, the treatments were compared on the basis of similar Brix; this comparison was possible because the untreated berries reached a Brix of 19.8 at $51 \mathrm{~d}$ while the CPPU-treated berries reached Brix of 19.6 at $70 \mathrm{~d}$. Comparing these two groups resulted in 313 and 589 DEGs with higher and lower expression from the untreated and CPPU-treated berries, respectively (Table S8). KEGG analysis demonstrated that in this 'similar Brix' group there was enrichment of 11 functional bins similar to those already present in cluster 3 (Table 2).

A subset of 42 genes showed significant differences in three combinations: (1) Control vs. CPPU at $51 \mathrm{~d}$; (2) Control vs. CPPU at $70 \mathrm{~d}$; (3) Control at $51 \mathrm{~d}$ vs. CPPU at $70 \mathrm{~d}$ (similar Brix) (Fig. 4d). Interestingly, very few DEGs had higher expression in the untreated berries at 
(a)
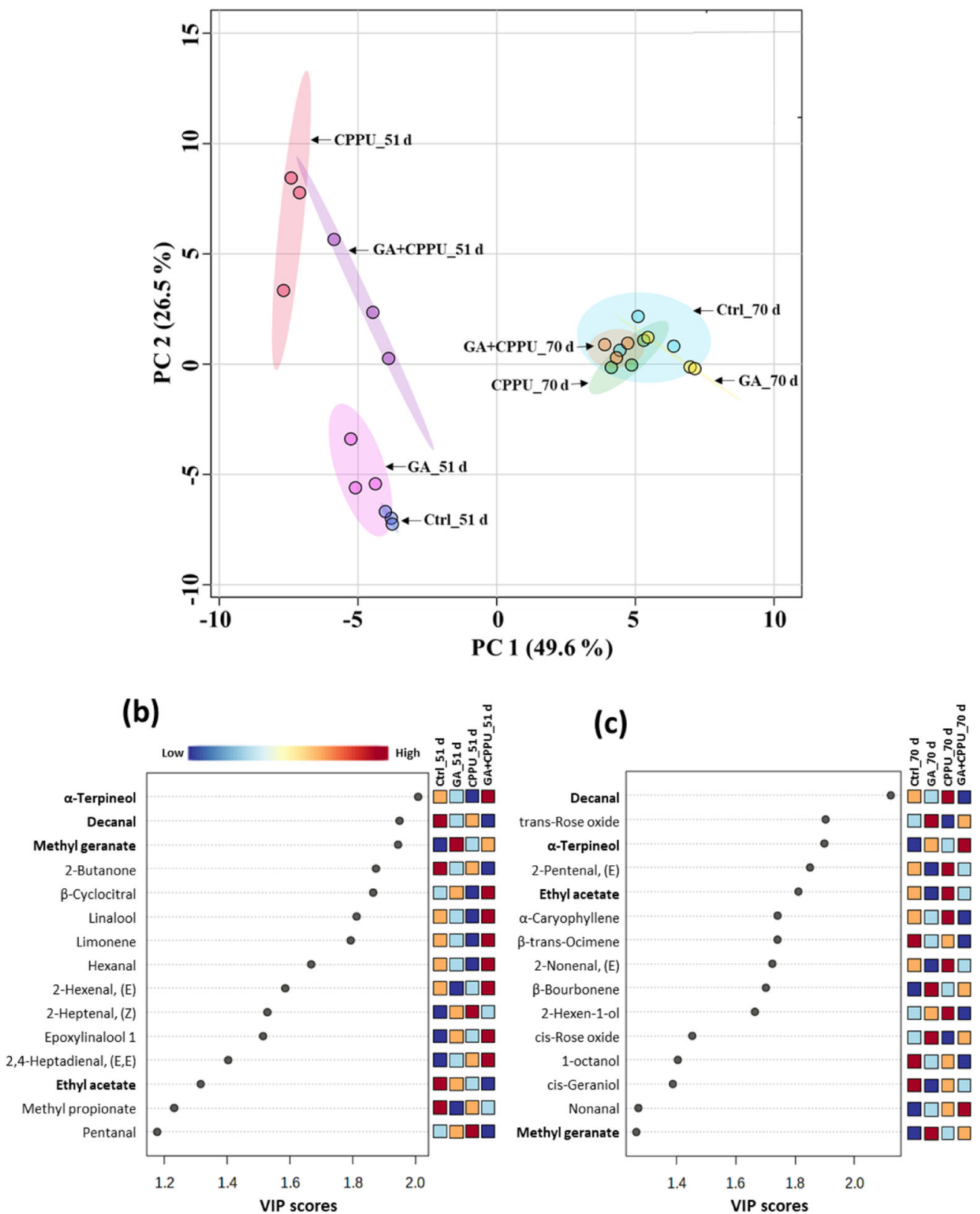

Fig. 3 Volatile content 'Sable' at $\mathbf{5 1}$ and $\mathbf{7 0} \mathbf{d}$ after growth regulator treatments. Volatile analysis of samples from GA-, CPPU-, and GA + CPPUtreated berries compared to the control was done by headspace SPME GC-MS analysis. a Principal component analysis (PCA) of volatile compounds was constructed for all treatments and time points. Variable importance in projection (VIP) scores of the variables at $51 \mathrm{~d}(\mathbf{b})$ and $70 \mathrm{~d}(\mathbf{c})$ for control, GA, CPPU, and GA + CPPU. The VIP score of a variable is calculated as a weighted sum of the squared correlations between the PLS-DA components and the original variable. Name in bold letters denotes common compounds (b) and (c). Color scale in (b) is applicable to (c) also

sampling of 51 and $70 \mathrm{~d}$. These genes were a germin-like protein, basic $7 S$ globulin (similar to xylanase inhibitor in Arabidopsis), protein P21 (similar to osmotin-like protein in Arabidopsis), polyol transporter 5 (similar to SIMPT4 in tomato expressed in late stages of fruit development), oleosin 1, and DMR6-like oxygenase 2 that functions in response to pathogens. The DEGs that had higher expression in response to CPPU could be assigned mainly to cell wall-related and stress-related functions. The cell wall-related DEGs included, in part, expansins, pectinesterase, pectate lyases, and NST1 that is considered a regulator of secondary cell wall 


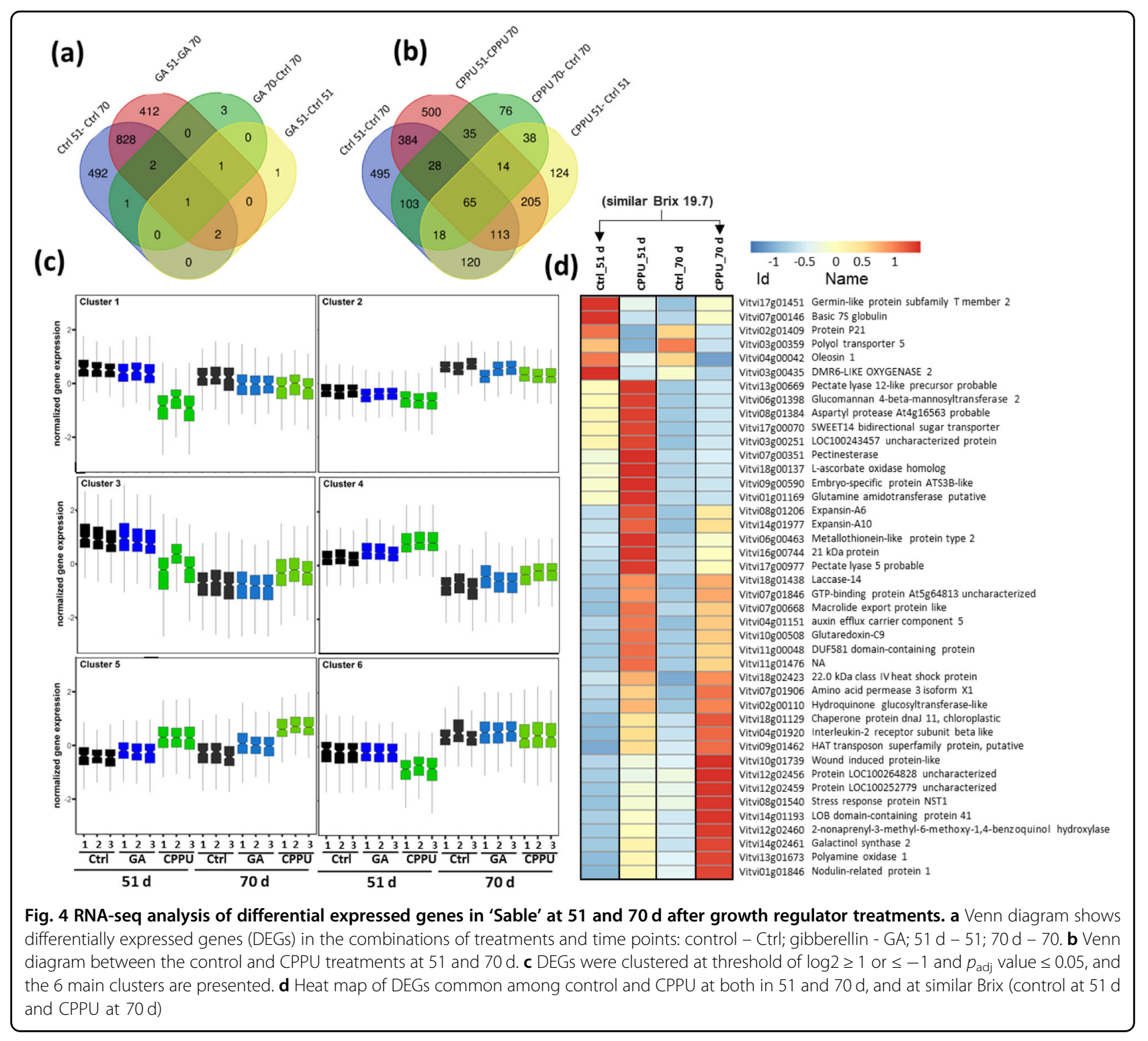

biosynthesis $^{29}$. The stress-related DEGs included the chaperone dnaJ 11, Nodulin-related protein 1, a metallothionein-like protein, and the hypoxia-associated genes $L O B$ domain-containing protein 41 and a 2-nonaprenyl-3-methyl-6-methoxy-1,4-benzoquinol hydroxylase.

The phenylpropanoid-related KEGG bins included $P A L$, trans-cinnamate 4monooxygenase, 4CL, caffeic acid $O$ methyltransferase, and cationic peroxidase 1 . The flavonoid bin included chalcone synthase (CHS) and the stilbenoid bin included stilbene synthases (STSs). The expression pattern of 56 DEGs related to the phenylpropanoid pathway that are significantly different among early control and late CPPU treatments (similar Brix group) is displayed in Fig. 5a. Interestingly, among the regulatory MYB genes 'MYB14, MYB15, and MYBA3' had lower expression following CPPU treatment, while three
MYB genes 'MYB136, MYB137, and MYB139' had higher expression in CPPU-treated at similar sugar levels. Of the WRKY family, 2 genes (WRKY22 and WRKY49) had higher expression while 5 genes (WRKY05, WRKY24, WRKY40, WRKY43, and WRKY51) had lower expression in CPPU-treated berries (Table S8). Of the structural genes $P A L, C 4 H, 4 C L, C H S, U F G T$, and remarkably, 28 STS genes, had lower expression in CPPU-treated berries (Fig. 5a). A scheme of the phenylpropanoid pathway with metabolites and genes affected by the CPPU treatment is shown in Fig. 5b.

The 'MAPK signaling' bin largely overlapped the 'Plant hormone signal transduction' bin. This hormone-related KEGG bin was enriched for the following genes: Auxin, AUX/IAA and SAUR; Cytokinin, A-ARR; Gibberellin, GID2; ABA, PYR/PYL and PP2C; Ethylene, ETR and 
Table 2 Process enrichment by KEGG analysis for DEGs belonging to cluster 3, cluster 4, or DEGs among early control and late CPPU treatment

\begin{tabular}{|c|c|c|c|c|c|c|}
\hline \multirow[t]{2}{*}{ KEGG Term } & \multirow[t]{2}{*}{ KEGG ID } & \multirow[b]{2}{*}{ Background $^{c}$} & \multicolumn{2}{|c|}{ All DEGs } & \multicolumn{2}{|c|}{ Control $51 \mathrm{~d} /$ CPPU $70 \mathrm{~d}^{\mathrm{f}}$} \\
\hline & & & Input $^{d}$ & $p$-Value ${ }^{\mathrm{e}}$ & Input & $p$-Value \\
\hline \multicolumn{7}{|l|}{ Cluster 3} \\
\hline Circadian rhythm - plant & vvi04712 & 72 & 20 & $8.01 \mathrm{E}-14$ & 25 & $1.79 \mathrm{E}-19$ \\
\hline Flavonoid biosynthesis & vvi00941 & 110 & 23 & $8.01 \mathrm{E}-14$ & 28 & 4.16E-19 \\
\hline Biosynthesis of secondary metabolites & vvi01110 & 1271 & 69 & $1.38 \mathrm{E}-11$ & 69 & 4.97E-12 \\
\hline Metabolic pathways & vvi01100 & 2390 & 88 & $1.32 \mathrm{E}-06$ & 87 & 9.65E-07 \\
\hline Phenylpropanoid biosynthesis & vvi00940 & 202 & 18 & $6.89 \mathrm{E}-06$ & 16 & $6.54 \mathrm{E}-05$ \\
\hline Phenylalanine metabolism & vvi00360 & 55 & 9 & $5.43 \mathrm{E}-05$ & 8 & 0.000237 \\
\hline Biosynthesis of secondary metabolites ${ }^{a}$ & vvi00999 & 15 & 5 & 0.000359 & 5 & 0.000237 \\
\hline Stilbenoid, diarylheptanoid and gingerol ${ }^{\mathrm{b}}$ & vvi00945 & 39 & 6 & 0.001958 & 9 & 3.74E-06 \\
\hline MAPK signaling pathway - plant & vvi04016 & 142 & 11 & 0.001958 & 10 & 0.004328 \\
\hline Ubiquinone and other terpenoid-quinone ${ }^{b}$ & vvi00130 & 43 & 6 & 0.002773 & - & - \\
\hline Phenylalanine, tyrosine and tryptophan ${ }^{b}$ & vvi00400 & 50 & 6 & 0.005204 & - & - \\
\hline Plant-pathogen interaction & vvi04626 & 236 & 13 & 0.008895 & - & - \\
\hline Plant hormone signal transduction & vvi04075 & 291 & 13 & 0.043811 & 13 & 0.034004 \\
\hline \multicolumn{7}{|l|}{ Cluster 4} \\
\hline Photosynthesis & vvi00195 & 21 & 21 & 2.65E-05 & & - \\
\hline Metabolic pathways & vvi01100 & 192 & 192 & 0.000653 & & - \\
\hline Biosynthesis of secondary metabolites & vvi01110 & 113 & 113 & 0.000744 & & - \\
\hline Photosynthesis - antenna proteins & vvi00196 & 7 & 7 & 0.010617 & & - \\
\hline Steroid biosynthesis & vvi00100 & 9 & 9 & 0.017040 & & - \\
\hline
\end{tabular}

Input/background number refer to the number of genes enriched for a specific process among all genes belonging to this process

anclassified

${ }^{b}$ Biosyntheiss

'Number of the genes in the term

${ }^{d}$ Number of genes enriched

${ }^{\text {e}}$ Corrected $p$-value

fSimilar Brix group

ERF1/2; Brassinosteroids, BAK1 and TCH4; and Jasmonic acid, JAZ (Fig. S2). Other enriched genes in the MAPK signaling included WRKY33, MKK9, and CaM4.

\section{Discussion}

Gibberellin has been used to enlarge seedless grape berries for many years ${ }^{9}$. Some cultivars are very sensitive to the treatment while others have a modest response ${ }^{10}$. Sable Seedless can be assigned to the category of 'modest response to GA', with a significant effect on size at early sampling that is reduced at late harvest. The transcriptomic data for both 51 and $70 \mathrm{~d}$ after the treatment, corresponding to early and late commercial harvest, suggest that GA did not have long-term effects on biological processes and this was also confirmed in the profile of phenylpropanoid metabolites. However, GA did delay maturity as expressed by lower TSS and TA (Fig. 1b, d), and also had an effect on volatile compounds (Table S3). In addition, when combined with CPPU, GA had some additive effects on fruit weight but it mitigated the response to CPPU with respect to acidity and reduction in some phenylpropanoid metabolites (Figs. 1d and 2). It is likely that higher doses of GA than the concentration used in this study $\left(20 \mathrm{mg} \mathrm{L}^{-1}\right)$, but still considered in the range of commercial practice for this cultivar, or multiple applications, would further increase the size and delay the ripening as previously reported in many studies ${ }^{30}$. Our data and the literature support the notion that GA can trigger quantitative effects on berry size, but does not have a major impact on ripening processes under standard practice.

In contrast to GA, CPPU affected a wide range of phenotypic, biochemical, and molecular responses. Since the 


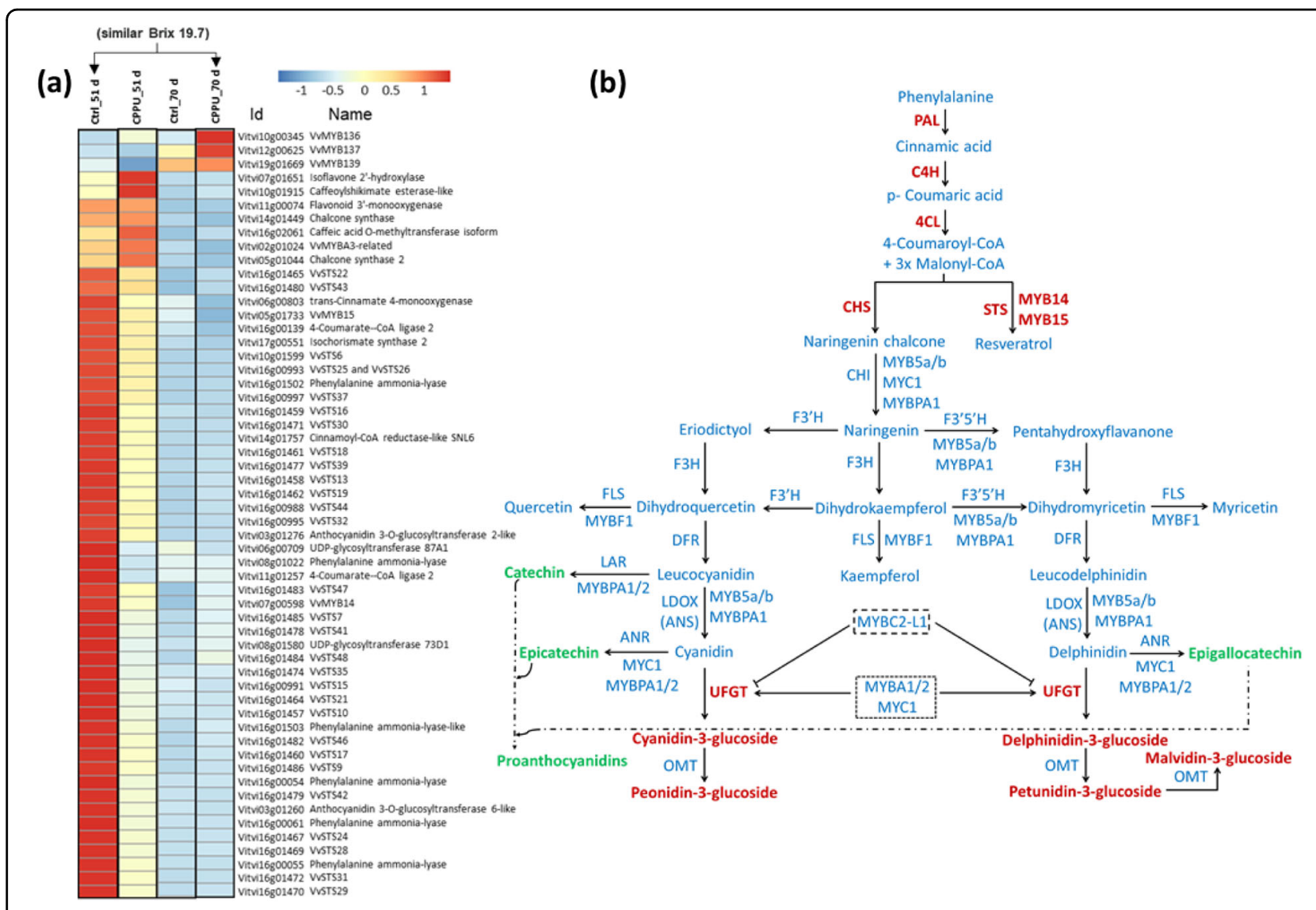

Fig. 5 Expression of phenylpropanoid-related genes and their position on the phenylpropanoid pathway. a Heat map of phenylpropanoidrelated DEGs among control and CPPU treatment at $51 \mathrm{~d}$, control and CPPU at $70 \mathrm{~d}$, and control at $51 \mathrm{~d}$ and CPPU at $70 \mathrm{~d}$ having similar ${ }^{\circ}$ Brix (indicated by arrow). $\mathbf{b}$ A scheme of the phenylpropanoid pathway with metabolites and genes affected by the CPPU treatment. The metabolites and genes in blue letters on pathway are not detected or remain unchanged. Green or red colored letters denote metabolites or gene up-regulated and down-regulated, respectively. PAL, Phenylalanine ammonia-lyase; $\mathrm{C4H}$, cinnamate 4-hydroxylase; 4CL, 4-Coumarate-CoA ligase; CHS, chalcone synthase; $\mathrm{CHI}$, chalcone isomerase; F3H, flavanone 3-hydroxylase; F3'H, flavonoid 3'-hydroxylase; F3' $5^{\prime} \mathrm{H}$, flavonoid 3'5'-hydroxylase; DFR, dihydroflavonol 4-reductase; LDOX, leucoanthocyanidin dioxygenase; UFGT, UDP glucose:flavonoid 3-O-glucosyltransferase; OMT, Omethyltransferase; FLS, flavonol synthase; LAR, leucoanthocyanidin reductase; ANR, anthocyanidin reductase. Panel (b) was modified from Kuhn et al. ${ }^{4}$

introduction of CPPU, there have been a number of studies in grapes reporting beneficial effects on fruit size, and delays in maturity, abscission, rachis browning, decay, cuticle formation, volatile profile and gene expression ${ }^{8,15-18,31}$. Our study on the colorless cultivar 'Thompson Seedless' pointed to the effect of CPPU on total tannin content resulting in higher astringency ${ }^{22}$. It was therefore of interest to study how the interplay between the anthocyanin branch and the PA branch of the pathway is manifested in colored cultivars. Our data clearly shows that synthesis of PA is at the expense of anthocyanins with no particular effects on the type of anthocyanins produced (Fig. 2a and Table S1). The fact that the level of flavonols was also reduced points to the possibility that either PA synthesis was induced by CPPU or that there was multiple repression of both the flavonol and anthocyanidin branches by genes such as $M y b A 1$ and $M y b F 1^{32}$. One interesting trend was the increase of (-)-epicatechin during late ripening, both in the control and CPPU-treated berries (Fig. 2c), in contrast to previous results showing that flavan-3-ols in the skins peak at 2-3 weeks after veraison ${ }^{33}$. This increase can result from degradation of PAs, but there was no evidence for this in the total PA levels and the only PA monomer that decreased in content during ripening was ECG (Fig. S1). Both flavan-3-ols, (+)-catechin and (-)-epicatechin, had higher concentration in CPPU-treated grapes and, thus, it can be hypothesized that there was a concomitant induction of their biosynthetic genes, $L A R$ and $A N R$ by TFs such as $M y b P A 1$ and $M y b P A 2^{34,35}$. CPPU did not affect the expression level of $V v M y b P A 1$ at $51 \mathrm{~d}$ but the level of this gene was reduced at $70 \mathrm{~d}$ post treatment (Table S7). It is not known if the gene was actually induced by CPPU before veraison. It should however be noted that PAs are synthesized prior to veraison while anthocyanins are synthesized 
during or after veraison, bringing up the possibility that the decrease in anthocyanins may be due to deficiency in substrates in the berry rather than repression of the ratelimiting genes. In previous studies, cytokinins were shown to promote anthocyanin synthesis in shoots and leaves of Arabidopsis with concomitant transcriptional activation of the pathway ${ }^{36}$. Likewise, introduction of a bacterial ipt gene to tobacco leaves increased the level of phenolic compounds $^{37}$.

The inherent discrepancy of comparing gene expression by treatments that delay maturity was partially compensated by comparing differential expression of phenylpropanoid-related genes at similar Brix, i.e., control at $51 \mathrm{~d}$ and CPPU at $70 \mathrm{~d}$ after treatment (Fig. $4 \mathrm{~d}$ ). There is a remarkable reduced expression of 28 STSs in CPPUtreated berries (Fig. 5a). The STS multigene family in grapes includes 48 annotated genes ${ }^{38}$. Expression maps of STS genes suggest induction of gene expression by multiple biotic stresses including ethylene and jasmonate, while abiotic stresses had both suppressive and inductive effects $^{39}$. Therefore, according to our data cytokinin may contribute to long-term inhibition of VvSTS expression. Of the upstream genes in the phenylpropanoid pathway, 6 DEGs corresponded to $P A L$ pointing to a trend of overall reduction in the pathway during ripening. Other genes included C4H, 4CL, CHS, UFGT (2 DEGs each), and 9 other structural genes of the pathway. Of the related transcription factors, MYB14 and MYB15 are considered as activators of STS gene expression ${ }^{39,40}$ and their low level is in agreement with the low expression of the STS genes. The expression of MYB14 and MYB15 was shown to increase with ripening in Pinot Noir grapes ${ }^{41}$, and therefore delay in ripening mediated by CPPU may reduce their expression. $M y b A 3$ was shown to be a truncated non-functional protein $^{42}$. MYB136, MYB137, and $M Y B 139$ showed high expression in CPPU-treated berries, and it will be interesting to find out if they are targets for cytokinin signaling in plants and bona fide regulators of VvSTS genes. Of the WRKY genes, WRKY24 and WRKY43 are reported to be co-expressed with STS and MYB genes ${ }^{39}$. For example, MYB14 and MYB15 were coexpressed with STS21, STS29, STS41, and STS48 in agreement with our data (Fig. 5a). Like MYB14 and $M Y B 15$, WRKY24 alone was shown to interact directly with the promoter of STS29.

A previous study on the effect of CPPU in 'Shine Muscat' grapes focused on transcriptome changes $60 \mathrm{~d}$ after the treatment and volatile profile changes $80 \mathrm{~d}$ after the treatment (i.e., when the berries reached very high maturity $)^{31}$. Similar to the previous report, our data showed that some genes of the phenylpropanoid pathway, e.g., PAL, $4 C L$, and UFGT had lower expression following CPPU treatment as well as fatty acid biosynthesis genes, amino acid biosynthesis, and volatile-related processes.
Higher expression was observed for carotenoid-related genes, fatty acid metabolism genes, and auxin-related genes. In hormonal signaling, Wang et $\mathrm{al}^{31}$ identified three accessions for TIFY $5 \mathrm{~A}$, two of which had higher expression at CPPU application level of $5 \mathrm{mg} \mathrm{L}^{-1}$ but with reduced expression at a level of $10 \mathrm{mg} \mathrm{L}^{-1} \mathrm{CPPU}$. This is consistent with our study where one of the TIFY $5 \mathrm{~A}$ had increased expression at both 51 and $70 \mathrm{~d}$ (Tables S6 and S7). Another set of affected hormone-signaling genes in our study was that of the cytokinin receptor genes, of which $A R R-5$ was repressed by CPPU at the late stage (Table S7). In contrast, in 'Shine Muscat' these genes were induced by CPPU. With respect to the volatile profile, similar reduction in terpenoids was encountered following CPPU treatment with respect to our sampling at $51 \mathrm{~d}$ (Fig. 3b). Several volatile compounds that were reduced after CPPU treatment in our study are consistent with reductions reported in Shine Muscat by Wang et al. ${ }^{31}$, i.e., $\alpha$-terpineol, linalool, 2-hexenal (E) at $51 \mathrm{~d}$ post treatment and cis geraniol at $70 \mathrm{~d}$ post treatment. Wang et al. ${ }^{31}$ also reported an increase in hexanol in 'Shine Muscat' following CPPU treatment but this was not the case for 'Sable' in our study (Table S3). Instead, we observed that CPPU increased other fatty acid derivatives such as decanal, 2-pentenal (E) and 2-hexen-1-ol at $70 \mathrm{~d}$ (Fig. 3c). Another important effect of CPPU in our study was the reduction in sesquiterpenes at $51 \mathrm{~d}$ (Table S3). However, the significance of this reduction is unclear given the fact that CPPU had no effect on the level of sesquiterpenes at $70 \mathrm{~d}$. The fact that GA increased the levels of rose oxide, $\alpha$-terpineol, and methyl geranate can strengthen the fruity flavor of the berries?

Given the large number of genes affected by CPPU, it was of interest to further look at a subset of genes that were consistently affected by the treatment at both 51 and $70 \mathrm{~d}$ after treatment and in the 'similar Brix' group (51 d control and $70 \mathrm{~d}$ CPPU). Two main processes were observed: increases in stress-related DEGs with hypoxia as a potential driver and increases in cell wall-related processes potentially associated with cell expansion (Fig. 4d). While there are no physiological studies to support it in our data, hypoxia has previously been associated with ripening and cell death in grape berries ${ }^{43}$ and it can be hypothesized that induction of stress/hypoxia-related genes by CPPU can therefore delay ripening. Mitigation of oxidative stress was attributed to cytokinin by proteins such as glutaredoxin (Fig. $4 \mathrm{~d})^{44}$. With respect to cell wallrelated genes, induction of expansins was described as a hallmark of response to cytokinin ${ }^{44}$ in addition to laccases and pectin-modifying genes (Fig. 4d).

There are multiple interactions among plant hormones and changes during ripening. When compared on the basis of similar Brix among the control and the CPPU treatment, it appears that there is repression of several key 
genes related to hormone signaling (Fig. S2). For example, $A U X / I A A$ genes are inhibitors of auxin signaling that is known to delay ripening ${ }^{45}$ while GID2 inhibits DELLA activity and allows response to gibberellins ${ }^{46}$. Other genes with reduced expression upon CPPU treatment were a $B R I 1$-associated kinase that controls brassinosteroids signaling and $J A Z$ that is a negative regulator of JA signaling ${ }^{5}$. Understanding the complex interaction between external hormone treatment and internal hormone signaling modules requires integration of knowledge from model systems with well-defined genetic resources and physiological data from crop plants.

At the scientific level, this study provides strong evidence for the induction of PA biosynthesis by cytokinin and to its effects on delay of ripening. On the other hand, GA is shown to influence berry size but it does not seem to have a major impact on metabolic and molecular processes including PA biosynthesis. At the practical level, information from this study can be used by growers to better understand how applications of PGRs can modulate berry size, taste, flavor, and color.

\section{Acknowledgements}

This work was funded by a United States-Israel Binational Agricultural Research and Development Fund Grant (No. IS-4957-16R). We thank the grower Asaph Arad for helping us with the vineyard experiments. We also thank Hanita Zemach for the help with the microscopy studies. The sequencing was carried out at the UC Davis Genome Center DNA Technologies and Expression Analysis Core, supported by NIH Shared Instrumentation Grant 1S10OD010786-01.

\section{Author details}

'Department of Postharvest Science, Agricultural Research Organization, The Volcani Center, Rishon LeZion, Israel. ${ }^{2}$ Department of Viticulture and Enology, University of California, Davis, CA 95616, USA. ${ }^{3}$ Department of Plant Pathology, Agricultural Research Organization, The Volcani Center, Rishon LeZion, Israel. ${ }^{4}$ Food Safety and Measurement Facility, University of California, Davis, CA 95616, USA

\section{Conflict of interest}

The authors declare no competing interests.

Supplementary information The online version contains supplementary material available at https://doi.org/10.1038/s41438-021-00488-0.

Received: 26 September 2020 Revised: 5 January 2021 Accepted: 11 January 2021

Published online: 01 March 2021

\section{References}

1. McAtee, P., Karim, S., Schaffer, R. J. \& David, K. A dynamic interplay between phytohormones is required for fruit development, maturation, and ripening. Front. Plant Sci. 4, 79 (2013).

2. Conde, C. et al. Biochemical changes throughout grape berry development and fruit and wine quality. Food 1, 1-22 (2007).

3. Lavee, S. Effect of gibberellic acid on seeded grapes. Nature 185, 395-395 (1960).

4. Kuhn, N. et al. Berry ripening: recently heard through the grapevine. J. Exp. Bot. 65, 4543-4559 (2013).

5. Falchi, R. et al. The genomics of grape berry ripening. In The Grape Genome (eds. Cantu, D. \& Walker, A.) 247-274 (Springer Nature, 2019).
6. Ebeler, S. E. \& Thorngate, J. H. Wine chemistry and flavor: looking into the crystal glass. J. Agric. Food Chem. 57, 8098-8108 (2009).

7. Maoz, I. et al. Insights into the chemosensory basis of flavor in table grapes. J. Sci. Food Agric. 100, 1405-1417 (2020).

8. Peppi, M. C. \& Fidelibus, M. W. Effects of forchlorfenuron and abscisic acid on the quality of 'Flame Seedless' grapes. HortScience 43, 173-176 (2008).

9. Weaver, R. \& McCune, S. Effect of gibberellin on seedless Vitis vinifera.Hilgardia 29, 247-275 (1959).

10. Acheampong, A. K. et al. Abnormal endogenous repression of GA signaling in a seedless table grape cultivar with high berry growth response to GA application. Front. Plant Sci. 8, 850 (2017).

11. Acheampong, A. K. et al. Functional characterization and developmental expression profiling of gibberellin signalling components in Vitis vinifera. J. Exp. Bot. 66, 1463-1476 (2015).

12. Giacomelli, L. et al. Gibberellin metabolism in Vitis vinifera L. during bloom and fruit-set: functional characterization and evolution of grapevine gibberellin oxidases. J. Exp. Bot. 64, 4403-4419 (2013).

13. Blank, R. et al. Effect of a forchlorfenuron dip on kiwifruit fruit size. N. Zeal. J. Crop Hort. Sci. 20, $73-78$ (1992).

14. Stern, R. A., Ben-Arie, R., Neria, O. \& Flaishman, M. CPPU and BA increase fruit size of 'Royal Gala'(Malus domestica) apple in a warm climate. J. Hortic. Sci. Biotech. 78, 297-302 (2003).

15. Reynolds, A., Wardle, D., Zurowski, C. \& Looney, N. Phenylureas CPPU and thidiazuron affect yield components, fruit composition, and storage potential of four seedless grape selections. J. Am. Soc. Hortic. Sci. 117, 85-89 (1992).

16. Zoffoli, J. P., Latorre, B. A. \& Naranjo, P. Preharvest applications of growth regulators and their effect on postharvest quality of table grapes during cold storage. Postharvest Biol. Technol. 51, 183-192 (2009).

17. Carvajal-Millán, E. et al. Polyphenol oxidase activity, color changes, and dehydration in table grape rachis during development and storage as affected by N-(2-chloro-4-pyridyl)-N-phenylurea. J. Agric. Food Chem. 49, 946-951 (2001).

18. Zabadal, T. J. \& Bukovac, M. J. Effect of CPPU on fruit development of selected seedless and seeded grape cultivars. Hortscience 41, 154-157 (2006).

19. Weaver, R., Van Overbeek, J. \& Pool, R. Effect of kinins on fruit set and development in Vitis vinifera. Hilgardia 37, 181-201 (1966).

20. Vogt, T. Phenylpropanoid biosynthesis. Mol. Plant. 3, 2-20 (2010).

21. Constabel, C. Molecular controls of proanthocyanidin synthesis and structure: prospects for genetic engineering in crop plants. J. Agric. Food Chem. 66, 9882 (2018).

22. Maoz, I. et al. Effect of the cytokinin forchlorfenuron on tannin content of Thompson Seedless table grapes. Am. J. Enol. Viticult. 65, 230-237 (2014).

23. Tyagi, K. et al. Girdling of table grapes at fruit set can divert the phenylpropanoid pathway towards accumulation of proanthocyanidins and change the volatile composition. Plant Sci. 296, 110495 (2020).

24. Coombe, B. G. Growth stages of the grapevine: adoption of a system for identifying grapevine growth stages. Aust. J. Grape Wine Res. 1, 104-110 (1995).

25. Ruzin, S. E. Plant Microtechnique and Microscopy. Vol. 198, $93-95$ (Oxford University Press, 1999).

26. Maoz, I., Beno-Moualem, D., Kaplunov, T., Lewinsohn, E. \& Lichter, A. Uneven distribution of flavour components in tablegrape berries. Aust. J. Grape Wine Res. 22, 343-349 (2016).

27. Tyagi, K. et al. Enhancement of table grape flavor by postharvest application of monoterpenes in modified atmosphere. Postharvest Biol. Technol. 159, 111018 (2020).

28. Chong, J., Wishart, D. S. \& Xia, J. Using MetaboAnalyst 4.0 for comprehensive and integrative metabolomics data analysis. Curr. Protoc. Bioinformatics 68, e86 (2019).

29. Mitsuda, N. et al. NAC transcription factors, NST1 and NST3, are key regulators of the formation of secondary walls in woody tissues of Arabidopsis. Plant Cell $19,270-280$ (2007)

30. Reynolds, A., Robbins, N., Lee, H.-S. \& Kotsaki, E. Impacts and interactions of abscisic acid and gibberellic acid on sovereign Coronation and Skookum seedless table grapes. Am. J. Enol. Viticult. 67, 327-338 (2016).

31. Wang, W., Khalil-Ur-Rehman, M., Feng, J. \& Tao, J. RNA-seq based transcriptomic analysis of CPPU treated grape berries and emission of volatile compounds. J. Plant Physiol. 218, 155-166 (2017).

32. Czemmel, S. et al. The grapevine R2R3-MYB transcription factor VuMYBF1 regulates flavonol synthesis in developing grape berries. Plant Physiol. 151, 1513-1530 (2009). 
33. Downey, M. O., Harvey, J. S. \& Robinson, S. P. Analysis of tannins in seeds and skins of Shiraz grapes throughout berry development. Aust. J. Grape Wine Res. 9, 15-27 (2003).

34. Bogs, J., Jaffé, F. W., Takos, A. M., Walker, A. R. \& Robinson, S. P. The grapevine transcription factor VvMYBPA1 regulates proanthocyanidin synthesis during fruit development. Plant Physiol. 143, 1347-1361 (2007).

35. Terrier, N. et al. Ectopic expression of VvMybPA2 promotes proanthocyanidin biosynthesis in grapevine and suggests additional targets in the pathway. Plant Physiol. 149, 1028-1041 (2009).

36. Deikman, J. \& Hammer, P. E. Induction of anthocyanin accumulation by cytokinins in Arabidopsis thaliana. Plant Physiol. 108, 47-57 (1995).

37. Schnablová, R. et al. Transgenic ipt tobacco overproducing cytokinins overaccumulates phenolic compounds during in vitro growth. Plant Physiol. Biochem. 44, 526-534 (2006).

38. Vannozzi, A., Dry, I. B., Fasoli, M., Zenoni, S. \& Lucchin, M. Genome-wide analysis of the grapevine stilbene synthase multigenic family: genomic organization and expression profiles upon biotic and abiotic stresses. BMC Plant Biol. 12 130 (2012).

39. Vannozzi, A. et al. Combinatorial regulation of stilbene synthase genes by WRKY and MYB transcription factors in grapevine (Vitis vinifera L.). Plant Cell Physiol. 59, 1043-1059 (2018).
40. Höll, J. et al. The R2R3-MYB transcription factors MYB14 and MYB15 regulate stilbene biosynthesis in Vitis vinifera. Plant Cell 25, 4135-4149 (2013).

41. Wong, D. C. J. et al. A systems-oriented analysis of the grapevine R2R3-MYB transcription factor family uncovers new insights into the regulation of stilbene accumulation. DNA Res. 23, 451-466 (2016).

42. Walker, A. R. et al. White grapes arose through the mutation of two similar and adjacent regulatory genes. Plant J. 49, 772-785 (2007).

43. Xiao, Z., Rogiers, S. Y., Sadras, V. O. \& Tyerman, S. D. Hypoxia in grape berries: the role of seed respiration and lenticels on the berry pedicel and the possible link to cell death. J. Exp. Bot. 69, 2071-2083 (2018).

44. Brenner, W. G., Ramireddy, E., Heyl, A. \& Schmülling, T. Gene regulation by cytokinin in Arabidopsis. Front. Plant Sci. 3, 8 (2012).

45. Böttcher, C., Harvey, K., Forde, C., Boss, P. \& Davies, C. Auxin treatment of preveraison grape (Vitis vinifera L.) berries both delays ripening and increases the synchronicity of sugar accumulation.Aust. J. Grape Wine Res. 17, 1-8 (2011).

46. Nelson, S. K. \& Steber, C. M. Gibberellin hormone signal perception: downregulating DELLA repressors of plant growth and development. Annu. Plant Rev. 1, 153-187 (2018) 